\title{
Optimization on Virtualization Network Laboratory Construction Security
}

\author{
Jun Lyn \\ Wuhan Technology and Business University, Hubei Wuhan 430223, China
}

Keywords: Virtualization, network lab, virtual experiment environment, remote practice

\begin{abstract}
With virtualization closed based planning in a single hardware environment for people to practice environment, in order to provide a laboratory which can let students use in the practical environment with limited hardware resource. Through the construction requirements of the laboratory and the characteristics of students' use, the problems of the traditional entity network safety laboratory are summarized and solved, and the feasibility of building the virtual laboratory is put forward. The development of the virtual laboratory in this way, allows students to have more than one network experimental environment and independent selectivity, with centralized management and automatic deployment of learning environment in the management, but also can solve the lack of experimental equipment space created by the laboratory for studying the problem of insufficient capacity. Finally, the conclusion that using virtual laboratory can effectively improve the teaching effectiveness of information security and network management is concluded.
\end{abstract}

\section{Introduction}

In recent years, information security has attracted more and more attention. In the education system, the training and education of network security personnel are becoming more and more important. However, in practice, practical operations are often needed to simulate, analyze and experiment real situations to enhance the management ability of network security. Therefore, to be able to provide a practical simulation of the real situation of the laboratory, education and training of I network security is very important, but in the face of cost and effectiveness under consideration, the construction of real information security laboratory often spend a lot of money, so the necessity of the network security laboratory more virtualization of its construction [1]. In the construction of a real network laboratory, the need for additional equipment to place space, run the required power consumption, hardware and software equipment maintenance, application deployment and a series of maintenance and management costs. In addition, from the perspective of laboratory management, the real experimental classroom cannot be open all-weather use, thus seriously affecting the use of the laboratory.

According to IDC (International Data Corporation) in the investigation of the virtual enterprise's global demand for 2013, at present the enterprise virtualization technology has two main applications, one is the real host virtualization allows the processor to effectively utilize resource and reduce the number of host enterprises, another is a storage device virtualization. This can make full use of storage space. Organizations and organizations that deploy virtualization will need software and hardware support, consultancy, enterprise integration and education and training services. It is expected that the market for global virtualization services will exceed $\$ 90$ billion by 2018 . When buying a new host needs, in order to improve the existing mainframe computing ability and save room space, or to the overall resource equipment planning, will use virtualization host or application virtualization.

\section{Related Technologies of Virtualization}

With the promotion of experimental teaching content, massive data applications need to be migrated to the experimental teaching platform. The problems of heterogeneous network, static 
resources, management complexity and high cost become important breakthroughs in the wave of a new round of educational reform. This paper proposes an open laboratory management system based on virtualization technology. Server virtualization, the intelligence of network devices and other technologies are applied to achieve open and intelligent management. The computing resources are optimized in the cloud computing environment and the clients are minimized. Thus, teachers and students can enjoy more quality experimental teaching services [3].

$$
\mathrm{C}_{k}=\left\{\begin{array}{lc}
\sqrt{1 / N} & k=0 \\
\sqrt{2 / N} & k=1,2, \cdots, N-1
\end{array}\right.
$$

With fully virtualized processors, full virtualization capabilities are available to support multiple systems without the need for additional modifications. Use Lin virtualized virtual host to install Linu, and Windows Server.

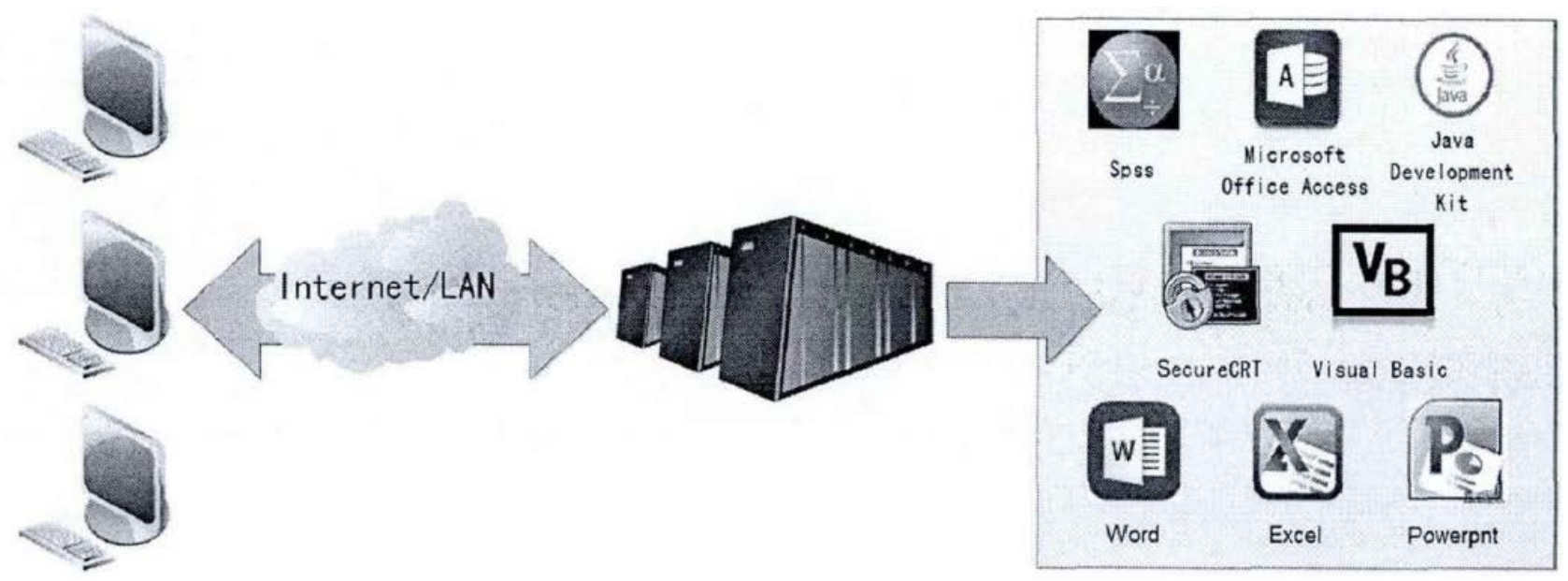

Fig. 1 Application virtualization architecture

We believe that the purpose of the internship is to be familiar with the server system environment. Only the use of virtualization is not in line with the internship requirements, so the amount of environment that the system can supply can be upgraded for this purpose. We have been in the Xen virtualization in Linux system; using Open VZ virtualization to improve the number of virtual environment system, to meet the needs of teaching, through the Open VZ, system virtualization, can provide a large number of experimental environment for practical use.

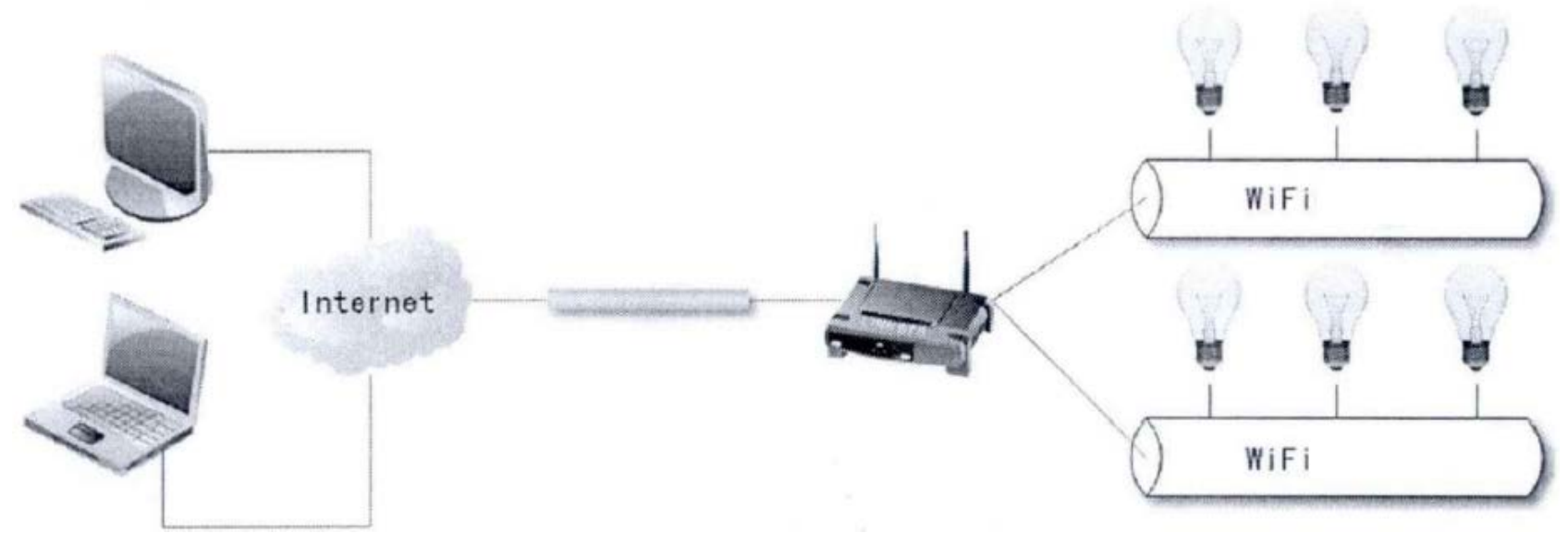

Fig. 2 Diagram of bench power supply control unit

As a result, the architecture diagram of Open VZ virtualization can provide network security practice for many people in a single hardware environment.

The connection through the establishment of VPN student practice, made to a server network address, the student will set software to board administrators created by the host, the use of SSH with 
the environment as independent of the hardware environment.

According to IDC (International Data Corporation) in the investigation of the virtual enterprise's global demand for 2013, at present the enterprise virtualization technology has two main applications, one is the real host virtualization allows the processor to effectively utilize resource and reduce the number of host enterprises, another for virtual storage devices so, can make full use of storage space. Organizations and organizations that deploy virtualization will need software and hardware support, consultancy, enterprise integration and education and training services. It is expected that the market for global virtualization services will exceed $\$ 90$ billion by 2018 . When buying a new host needs, in order to improve the existing mainframe computing ability and save room space, or to the overall resource equipment planning, will use virtualization host or application virtualization.

\section{Security Laboratory Management System Based on Virtualization Technology}

First of all, a large cloud platform that is suitable for massive data computing and storage is built. And the user data, application software are deployed in the server cluster. Parallel and distributed computing technology is applied to provide users with high-performance services. The thin clients replace the host, which reduced the cost of laboratory construction greatly and improved the scalability of hardware devices. Video redirection technology is used to ensure the effect of client video playback. Using, the video files are sent to the thin client directly and decoded by the ARM hardware for playback, which reduced the occupation of the server CPU. In order to achieve the same user experience of cloud desktop with the PC. RDP8.0 efficient delivery protocol, detection of the network status, adaptive graphics and media flow are adopted to improve the transmission efficiency.

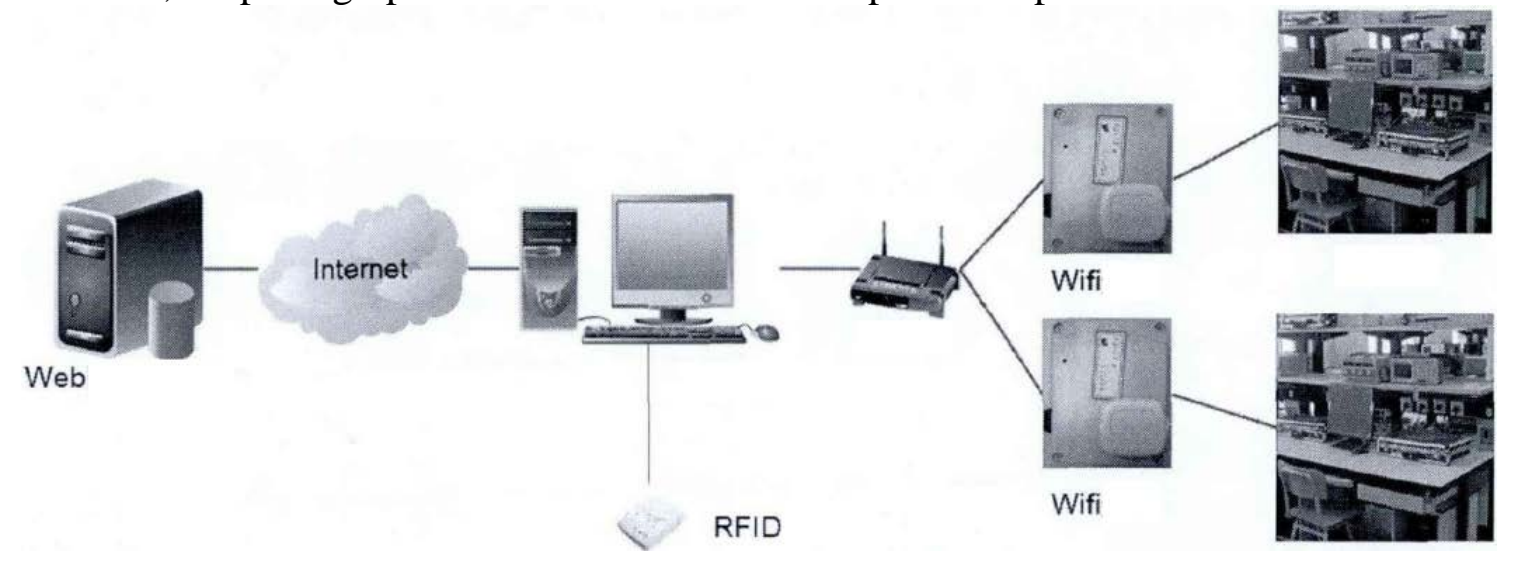

Fig. 3 Diagram of hardware system

Second, the open laboratory management system website are designed and implemented. The features of this system include: equipment information management, resource statistics, booking management, curriculum management, user management, etc. The system not only achieves the basic information of electronic management, but also provides students with an integrated experimental booking service and guidance. Moreover, it provides scheduling function of experiment courses for teachers. The problem of scheduling is an NP-complete problem (the non-deterministic problem of polynomial complexity), and the complexity of the experimental class arrangement is higher than that of the general theory course. Therefore, a combination of automatic scheduling algorithm uses the priority search and comparison to replace the shortest path rule, which simplifies the search level and reduces the spatiotemporal complexity of the scheduling algorithm.

$$
\begin{aligned}
& \mathrm{Y}_{\mathrm{mn}}=C_{m} C_{n} \sum_{i=0}^{N-1} \sum_{j=0}^{N-1} X_{i j} \cos \frac{(2 j+1) n \pi}{2 N} \cos \frac{(2 i+1) m \pi}{2 N} \\
& \mathrm{X}_{m n}=\sum_{i=0}^{N-1} \sum_{j=0}^{N-1} C_{m} C_{n} Y_{m n} \cos \frac{(2 j+1) n \pi}{2 N} \cos \frac{(2 i+1) m \pi}{2 N}
\end{aligned}
$$


Finally, the wireless control terminals of device power is designed and implemented. AP nodes are distributed to each equipment. The intelligent control of the power supply of the equipment is realized arranging the Wi-Fi network. After the success of appointment, students will receive an SMS by SMS module automatically and swiping cards to turn on the computer by them. The client server will send commands to supply the device power.

Table 1 OpenVZ

\begin{tabular}{cccc}
\hline Number & 1 & 10 & 100 \\
\hline Create & $11.568 \mathrm{~s}$ & $2 \mathrm{~m} 31.254 \mathrm{~s}$ & $25 \mathrm{~m} 8.541 \mathrm{~s}$ \\
Start & $1.740 \mathrm{~s}$ & $6.573 \mathrm{~s}$ & $9 \mathrm{~m} 57.231 \mathrm{~s}$ \\
Stop & $0.769 \mathrm{~s}$ & $6.229 \mathrm{~s}$ & $1 \mathrm{~m} 16.825 \mathrm{~s}$ \\
Destory & $1.058 \mathrm{~s}$ & $9.877 \mathrm{~s}$ & $2 \mathrm{~m} 36.435 \mathrm{~s}$ \\
\hline
\end{tabular}

$\mathrm{VM}$ is an open source virtual machine monitor (Virtual Machine Monitor), is between the underlying hardware and operating system between the middleware layer (that is, Virtualization Layer), which provides abstraction of the underlying hardware resources, can be regarded as hardware architecture Extended, can shorten the upper virtual machine in the implementation of the additional burden (overhead), so can bring higher efficiency. Different virtualized environments have different performance, VM's virtual environment in general, only $2 \%$ of the loss, up to only $80 \%$, compared to the full virtualization software VM ware, the highest $20 \%$ of the loss.

\section{Summary}

With the increasing complexity of network environment, in a laboratory environment to achieve the degree of complexity is not easy, because the physical facilities construction costs are too high, so it can meet the reality such as the complexity of the network environment in the teaching environment construction, will enrich the teaching content, so the network security experiment environment virtualization does the necessity of construction.

The virtual server used in remote teaching environment, to reduce the overall cost, improve safety, but also can reduce the burden of management, and bring a variety of options for teaching, through a single hardware virtualization environment to achieve better density (virtualization density), flexibility (flexibility), portability (scalability), management (manageability), bring a new look for the laboratory practice.

In real experiment, the video transmission is smooth, and the thin client can receive video, trans code and play in time. When a client is added to play video, the server CPU, memory, occupied bandwidth and other indicators will increase by about $4.5 \%$. Each client takes about $1.4 \mathrm{Mbps}$ broadband and the network delay is less than 1.3s, which can meet the requirements.

\section{References}

[1] Wu-Jin F U. Construction of the Virtual Network Laboratory Based on Flexible Environment. (Computer Knowledge \& Technology, 2010), p. 1-6. (In Chinese)

[2] Feng L I, Guo G Q. Network Security Virtual Laboratory. (Computer Systems \& Applications, 2014), p. 120-124.

[3] Lu D, Chen L Q, Qian X Y D, et al. Network-Based Construction of Open Virtual Laboratory Teaching Platform (International Conference on Information Computing and Applications. Springer Berlin Heidelberg, 2011), p. 427-433.

[4] Iguchi N, Kurauchi M, Tsujimoto $\mathrm{Y}$, et al. IP network construction learning system utilizing virtual router. Vol. 10 (2011) No. 1, p. 107-112.

[5] Wang H, Du H, Xu G, et al. Construction of Network Virtual Laboratory. (Energy Procedia, 2011), No. 13, p. 6204-6209.

[6] Li P, Mohammed T. Integration of virtualization technology into network security laboratory (Frontiers in Education Conference, 2008. Fie 2008. IEEE, 2008), p. S2A-7 - S2A-12. 